\title{
Triple-Labeling Whole-Mount In Situ Hybridization Method for Analysis of Overlapping Gene Expression in Brain Tissue with High Level of Autofluorescence
}

\author{
Jie Chen ${ }^{1}$, Cindy Laramore ${ }^{1}$ and Michael Shifman ${ }^{1,2 *}$ \\ ${ }^{1}$ Shriners Hospital's Pediatric Research Center (Center for Neural Repair and Rehabilitation), USA \\ ${ }^{2}$ Department of Neuroscience, Temple University School of Medicine, 3500 North Broad Street, Philadelphia, PA 19140, USA
}

\begin{abstract}
Background: Detection of multiple co-localized mRNAs by conventional chromogenic in situ hybridization is difficult because the first reaction product can obscure subsequent ones. Multi-color fluorescent in situ hybridization (FISH) offers simultaneous detection of multiple mRNAs but has low sensitivity and in cells of the central nervous system (CNS), is hampered by high background autofluorescence.
\end{abstract}

New method: Our method of sequential triple colorimetric in situ hybridization in whole-mounted lamprey brain, which has high background autofluorescence, uses three Alkaline Phosphatase (AP) substrates - Fast Red, 2-(4-iodophenyl)-5-(4-nitrophenyl)-3-phenyltetrazolium chloride (INT) /5-bromo-4-chloro-3-indolyl-phosphate (BCIP) and nitro blue tetrazolium (NBT) /BCIP, to develop red, yellow-brown and purple-blue signals sequentially. To achieve sequential colorimetric in situ hybridization, we selected AP substrates that produce ethanol-soluble reaction products, such as Fast Red and INT/BCIP. The most important step is to wash away the current color before the next color is developed.

Results: Using this sequential triple colorimetric in situ hybridization method, we showed that the netrin receptors deleted in colorectal cancer (DCC) and uncoordinated 5 (UNC-5), as well as the repulsive guidance molecule (RGM) receptor Neogenin, are all co-expressed in some reticulospinal neurons of the larval lamprey brainstem. The sensitivity and specificity of the fluorescence method in whole-mounted lamprey brain, which has a high degree of autofluorescence, were greater than those of a commercial colorimetric method, the Tyramide Signal Amplification (TSA) Fluorescence Kit.

Conclusions: The non-fluorescent chromogenic method is a simple, sensitive and reliable alternative to FISH on whole-mounted tissue with strong endogenous autofluorescence. And this sequential non-fluorescent chromogenic method allows detection of three overlapping or co-localized mRNA targets without loss of sensitivity in the second and third rounds of detection, and without masking of lighter signals by stronger ones.

Keywords: In situ hybridization; Endogenous autofluorescence; Wholemounted brain

\begin{abstract}
Abbreviations: FISH: Fluorescent In Situ Hybridization; CNS: Central Nervous System; AP: Alkaline Phosphatase; INT: 2-(4-iodophenyl)5-(4-nitrophenyl)-3-phenyltetrazolium Chloride; BCIP: 5-bromo4-chloro-3-indolyl-phosphate; NBT: Nitro Blue Tetrazolium; DCC: Deleted in Colorectal Carcinoma; UNC-5: Uncoordinated 5; RGM: Repulsive Guidance Molecule; TSA: Tyramide Signal Amplification; DIG: Digoxigenin; HRP: Horseradish Peroxidase; BIO: Biotin; FITC: Fluorescein Isothiocyanate; GFP: Green Fluorescent Protein; MFB: Multiple Filter Block; SB: Sudan Black B; UV: Ultraviolet; PAF: Paraformaldehyde; PBS: Phosphate Buffered Saline; MB: Maleate Buffer; RT: Room Temperature; O/N: Overnight; POD: Peroxidase
\end{abstract}

\section{Introduction}

Whole-mount in situ hybridization is the method of choice for examining the expression patterns of developmentally regulated genes in embryos of Drosophila, Xenopus, zebrafish, medaka, sea lamprey, chickens and mice [1-4]. Whole-mounted preparations increase the amount of information gathered per experiment and preserve three-dimensional information, which allows the rapid and accurate identification of labeled cells. Faster and easier transcript visualization in whole-mounted tissues and embryos was achieved by introduction of nonradioactive labeling, in which anti-sense RNA probes are labeled with the small molecule digoxigenin (DIG) and are recognized by an anti-DIG antibody conjugated to alkaline phosphatase (AP). During incubation with the substrate nitro blue tetrazolium (NBT)/5-bromo4-chloro-3-indolyl-phosphate (BCIP), this enzyme deposits a brownish purple precipitate in cells. Subsequent improvements permitted detection of two or three different gene products, using different colors of chromogenic stains within the same sample. With this technique, fluorescein, biotin and digoxigenin probes were visualized using antibodies specific to the probe labels and conjugated with AP or horseradish peroxidase (HRP). Several combinations of enzyme/ chromogenic substrate were developed for chromogenic detection of in situ signal $[5,6]$. The combination used most often is anti-DIG antibody conjugated with AP and BCIP/NBT chromogenic substrate. This produces a good signal-to-noise ratio, very good sensitivity and good

*Corresponding author: Michael Shifman, Shriners Hospitals Pediatric Research Center (Center for Neural Repair and Rehabilitation) and Department of Neuroscience, Temple University School of Medicine, 3500 North Broad Street Philadelphia, PA 19140, USA, Tel: 1215 926-9321; Fax: +1 215-926-9325; E-mail: mshifman@temple.edu

\section{Received April 13, 2015; Accepted May 19, 2015; Published May 21, 2015}

Citation: Chen J, Laramore C, Shifman M (2015) Triple-Labeling Whole-Mount In Situ Hybridization Method for Analysis of Overlapping Gene Expression in Brain Tissue with High Level of Autofluorescence. J Cytol Histol S3:011. doi:10.4172/2157-7099.S3-011

Copyright: (c) 2015 Chen J, et al. This is an open-access article distributed under the terms of the Creative Commons Attribution License, which permits unrestricted use, distribution, and reproduction in any medium, provided the original author and source are credited. 
spatial resolution, often at a cellular level. However, a major limitation of these histochemical multi-labeling techniques is that overlapping regions of two expression patterns are difficult to discern. In cells where both reaction products are present, one product may obscure the presence of the other, depending on their relative abundance and color. In addition, overlapping or co-localized expression is often difficult to resolve because of lower sensitivity in the second and third rounds of detection.

In theory, these limitations can be circumvented by using multicolor fluorescent in situ hybridization (FISH), which can offer simultaneous detection of multiple target RNAs at high spatial resolution. With this technique, fluorescein, biotin and digoxigenin probes can be visualized using primary antibodies specific to the probe labels and secondary antibodies conjugated with brightly fluorescent dyes (Alexa or Cyanine). However, detection of low-abundance mRNA posed a challenge for conventional FISH, due to low sensitivity compared to chromogenic AP detection, because the latter can be amplified enzymatically [7]. Therefore, whole-mount FISH methods add a signal amplification step, using fluorescent tyramide substrates in sequential horseradish peroxidase reactions (tyramide signal amplification, TSA) [8-10]. A serious limitation of the TSA reaction is the rapid inactivation of horseradish peroxidase by substrate excess, so that the TSA reaction can last no more than 30 minutes. This often is not sufficient for detection of sparsely expressed transcripts [11,12]. By contrast, AP enzymatic activity can be carried out for several hours resulting in high signal-to-noise ratio.

Moreover, a major limitation the FISH method is the high background autofluorescence seen in marine animals, such as zebrafish embryos [13,14] and lampreys, and in the central nervous system (CNS) of most species (particularly in post-mitotic neurons and glial cells) [15]. Autofluorescence, either intrinsic or induced by fixation reagents and tissue processing, may mask specific fluorescent signals or be mistaken for fluorescent labels [16,17]. Autofluorescence of neural tissue often complicates the use of the green fluorescent protein (GFP) [18]. More importantly for our research, animals with spinal cord transection showed significant autofluorescence of cortical, brainstem, and spinal cord neurons, which interferes with identification of labeling $[19,20]$. In our own research, we have encountered unacceptable levels of autofluorescence in lamprey brain and spinal cord neurons after injury (Figure 1). Axotomy increases lipofuscin pigment in the trigeminal ganglion [21]. Lipofuscin has broad excitation and emission spectra $[15,22]$ that overlap those of almost all generally-used fluorochromes (except far-red), mimicking the appearance of immunofluorescent labeling and making it difficult or impossible to distinguish between specific labeling and nonspecific autofluorescence. Lipofuscin is a native autofluorescent material that persists even in paraffin sections. It can be especially annoying in certain large neurons in the CNS. Lipofuscin is brown in color and stains red by Sudan Black and is PAS-positive. Fluorescence properties: Excitation: UV-blue; Emission: green-yelloworange. Prominent in neurons, glial cells cardiac muscle cells but found in a wide range of cell types. Predominately post-mitotic cells. Lipofuschin has an enigmatic chemistry and stains positive for proteins, carbohydrates and lipids (!) [23]. Lipofuscin is the breakdown product of old red blood cells - an "aging" pigment. It usually occurs as small, punctate intracellular structures that are strongly fluorescent under any excitation ranging from $360 \mathrm{~nm}$ to $647 \mathrm{~nm}$. The color should appear orange under UV excitation, green or yellow under blue excitation, or red under green excitation.

Several methods have been described for reducing autofluorescence:
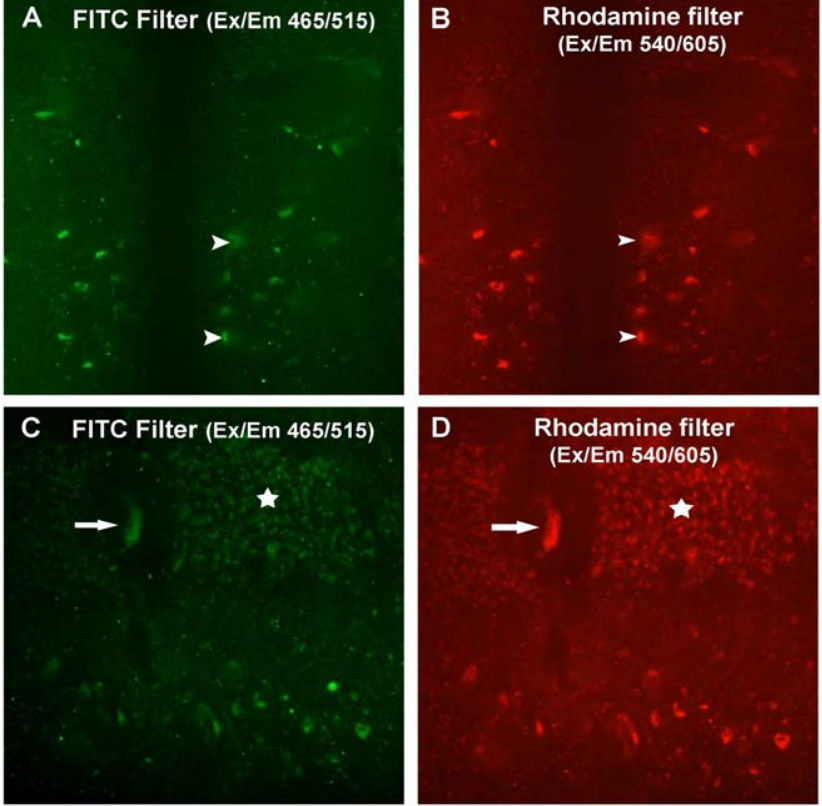

Figure 1: Endogenous autofluorescence in lamprey brainstem after spinal cord injury. (A) Background autofluorescence in larval lamprey brain reticulospinal neurons under $465 \mathrm{~nm}$ excitation wavelength Arrowheads point to neurons with autofluorescence. (B) The same brain area displays autofluorescence under $540 \mathrm{~nm}$ excitation wavelength. Arrowheads point to neurons with autofluorescence. (C) and (D) The nucleus of a lamprey brain neuron displaying strong, broad ( $465 \mathrm{~nm}-540$ $\mathrm{nm}$ ) autofluorescence. White arrows point to the nucleus; white stars are in an area small, autofluorescent neurons/glial cells.

histochemical techniques $\left(\mathrm{CuSO}_{4}\right.$ in ammonium acetate buffer or Sudan Black B (SB) in 70\% ethanol) [24]; $\mathrm{NaBH}_{4}$ [25], Pontamine Sky Blue [26]. a multiple filter block (MFB) strategy, in which neurons are examined with three different filter blocks and a neuron is considered labeled if it fluoresces with only one of them; photobleaching by irradiation with ultraviolet (UV) light before treatment with fluorescent probes [27]; and mathematical models that subtract the background autofluorescence from digital images on a pixel-by-pixel basis [28]. Each of these methods has disadvantages. Treatment of tissue with $\mathrm{CuSO}_{4}$ or SB unacceptably reduces the intensity of immunofluorescent labeling, and mathematical models are technically difficult and potentially inaccurate.

Therefore, we have developed a chromogenic triple-labeling wholemount in situ hybridization method that allows us to study overlapping gene expression patterns in single neurons and avoids the adverse effects of autofluorescence. This method is sensitive enough to detect the co-localization of three axon guidance molecule receptors, deleted in colorectal cancer (DCC), uncoordinated 5 (UNC-5) and repulsive guidance molecule (RGM) receptor Neogenin.

Our sequential triple colorimetric in situ hybridization method will allow us to capture images immediately after each staining step, then inactivate AP and wash the current color with ethanol before next round of antibody incubation and staining. This innovation allowed us to avoid one of main disadvantages of previously reported multicolor AP- based chromogenic in situ hybridization - inability to distinguished overlapping or co-localized gene expression patterns because they are difficult to differentiate visually when produced at the same site. Moreover, using our method it is much easy to control 
each step within an appropriate reaction time and to get a clean and clear expression view of each gene represented by separate color. Last consideration is particularly important when studying gene expression of low-abundance multiple molecules in single neurons.

\section{Materials and Methods}

\section{Animals}

All the experimental procedures with animals were approved by the Institutional Animal Care and Use Committee at Temple University. Wild-type larval lampreys (Petromyzon marinus), $12-14 \mathrm{~cm}$ in length (4-5 years old), were obtained from streams feeding Lake Michigan and maintained in fresh water tanks at $16^{\circ} \mathrm{C}$ under a 12 hour light cycle until the day of surgery. Animals were anesthetized in $0.1 \%$ tricaine methanesulfonate (Spectrum Chemical, Gardena, CA), and the brains were removed for in situ hybridization.

\section{Riboprobe synthesis}

Sequences of the lamprey netrin receptors DCC, UNC5 and the RGM receptor Neogenin (GenBank accession numbers AY744917, AF129475 and HM488345.1 respectively) were used for the generation of sense and antisense riboprobes. These sequences were used to construct PCR templates which included the T7 promoter sequence, and RNA probes were transcribed using T7 RNA polymerase (Promega, Madison, WI). DCC, UNC5 and Neogenin probes were labeled with digoxigenin (DIG), biotin (BIO) and fluorescein (FITC) respectively for the triple-labeling in situ hybridization. The incorporation of DIG, BIO and FITC into probes was controlled by dot blots. The length and integrity of the probes were examined by gel electrophoresis. Labeled sense RNA probes were used as controls.

\section{Lamprey brain tissue pretreatment and single alkaline phosphatase chromogenic in situ hybridization.}

For in situ hybridization, brains were dissected from anesthetized lampreys, stripped of choroid plexus, pinned flat to a small strip of Sylgard and fixed in $4 \%$ paraformaldehyde (PAF) -phosphate buffered saline (PBS) 2.5 - 3 hours at room temperature (RT). After washing in $\mathrm{PTw}\left(0.1 \%\right.$ Tween 20 in PBS) $3 \times 10 \mathrm{~min}$ at $4^{\circ} \mathrm{C}$, brains were prehybridized at $50^{\circ} \mathrm{C}$ in hybridization solution $(50 \%$ deionized formamide; $5 \mathrm{x} \mathrm{SSC} ; 100 \mu \mathrm{g} / \mathrm{ml}$ Torula yeast RNA; $100 \mu \mathrm{g} / \mathrm{ml}$ wheat germ t RNA; $50 \mu \mathrm{g} / \mathrm{ml}$ heparin; $0.1 \%$ Tween 20$) 1$ hour and then hybridized $24-36$ hours at $50^{\circ} \mathrm{C}$ in the same solution plus $1 \mu \mathrm{g} / \mathrm{ml}$ DIG-, BIO- or FITC-labeled RNAs. After washing in maleate buffer $+0.1 \%$ Tween 20 (MB) and 1 hour incubation in MB with 2\% Blocking Reagent (Roche, Indianapolis, IN), brains were incubated with AP -conjugated antiDIG, Streptavidin or anti-FITC antibodies (Roche) 1:1000 in maleate buffer $(+0.1 \%$ Tween 20$)$ with $2 \%$ Blocking Reagent $\mathrm{O} / \mathrm{N}$ at $4^{\circ} \mathrm{C}$. Next day samples were washed in maleate buffer $+0.1 \%$ Tween 20 and in Fast Red buffer (100 mM Tris-HCl, pH 8.2, ) or AP buffer $(100 \mathrm{mM} \mathrm{NaCl}$, $50 \mathrm{mM} \mathrm{MgCl}, 100 \mathrm{mM}$ Tris-HCl, pH 9.5, 0.1\% Tween 20). Finally, the probes were detected by different AP substrates - Fast Red, INT/BCIP or NBT/BCIP (Roche) chromogenic reaction in the dark at $37^{\circ} \mathrm{C}$ or RT. Reaction was stopped by washing in distilled water. After re-fixation with 4\% PAF in PBS and washing 3 times in $1 \mathrm{x}$ PBS for $10 \mathrm{~min}$, brains were mounted under glycerol and photographed.

\section{Sequential three-color chromogenic in situ hybridization in whole-mount lamprey brain}

DIG-DCC labeling: For sequential triple labeling in situ hybridization, we hybridized the brain samples simultaneously with
DIG-DCC, BIO-Neogenin and FITC-UNC5 RNA probes. After hybridization, brains were sequentially washed in hybridization solution at $55^{\circ} \mathrm{C}$, then in PTw and $\mathrm{MB}$ at RT. After tissues were blocked in $\mathrm{MB}$ with 2\% Blocking Reagent for $1 \mathrm{~h}$, alkaline phosphatase (AP) conjugated anti-DIG Fab (Roche; 1:1000) in blocking buffer was applied to tissue overnight at $4^{\circ} \mathrm{C}$. After several washes in $\mathrm{MB}$ and in $100 \mathrm{mM}$ Tris-HCl, pH 8.2, digoxigenin-labeled probe was detected by Fast Red chromogenic reaction for $3 \mathrm{~h}(\mathrm{RT})$, then $\mathrm{O} / \mathrm{N}$ at $4^{\circ} \mathrm{C}$. Reaction was stopped by washing in $100 \mathrm{mM}$ Tris-HCI, $\mathrm{pH}$ 8.2. Brains were mounted under glycerol and photographed. Before the second round of labeling, the antibody-AP conjugate was inactivated by $0.1 \mathrm{M}$ glycine- $\mathrm{HCl}(\mathrm{pH}$ 2.2) with $0.1 \%$ Tween 20 for $30 \mathrm{~min}$ at $\mathrm{RT}$ or $37^{\circ} \mathrm{C}$, and Fast Red stain was removed by sequential washes in $50 \%, 70 \%, 90 \%, 95 \%$ and $100 \%$ ethanol for 5 min each step.

BIO-Neogenin labeling: After washing in $\mathrm{MB}$ and incubating $1 \mathrm{~h}$ in blocking solution, brains were incubated with AP-conjugated streptavidin (Roche) 1:1000 in blocking solution $\mathrm{O} / \mathrm{N}$ at $4^{\circ} \mathrm{C}$. Next day they were washed in maleate buffer $+0.1 \%$ Tween 20 and in AP buffer (100 mM NaCl, $50 \mathrm{mM} \mathrm{MgCl} 2,100 \mathrm{mM}$ Tris-HCl, pH 9.5, 0.1\% Tween 20). Biotin-labeled probe was detected by INT/BCIP chromogenic reaction in the dark at $37^{\circ} \mathrm{C}$ or RT. Reaction was stopped by washing in $0.1 \mathrm{M}$ Tris-HCI, $\mathrm{pH}$ 8.2. Brains were mounted under glycerol and photographed. Before the third round of labeling, the antibody-AP conjugate was inactivated by incubation in $0.1 \mathrm{M}$ glycine- $\mathrm{HCl}(\mathrm{pH} 2.2)$ with $0.1 \%$ Tween 20 for $30 \mathrm{~min}$ at RT, and INT/BCIP stain was removed by sequential washes in $50 \%, 70 \%, 90 \%, 95 \%$ and $100 \%$ ethanol for 5 min each step.

FITC-UNC5 labeling: After washing in maleate buffer $+0.1 \%$ Tween 20 and $1 \mathrm{~h}$ incubation in maleate buffer $(+0.1 \%$ Tween 20$)$ with 2\% Blocking Reagent, brains were incubated with AP-conjugated antifluorescein antibody (Roche; $1: 1000)$ in maleate buffer $(+0.1 \%$ Tween 20 ) with $2 \%$ Blocking Reagent $\mathrm{O} / \mathrm{N}$ at $4^{\circ} \mathrm{C}$. Next day they were washed in maleate buffer $+0.1 \%$ Tween 20 and in AP buffer. Fluorescein -labeled probe was detected by NBT/BCIP chromogenic reaction in the dark on ice or RT. Reaction was stopped by washing in distilled water and then in 1x PBS ( $\mathrm{pH} 5.5$ ) in the dark. After samples were refixed with $4 \%$ PAF in $1 \times$ PBS for $30 \mathrm{~min}$, brains were mounted under glycerol and photographed with a Nikon Eclipse 80i microscope with attached digital camera using $2 \mathrm{x}, 4 \mathrm{x}$ and 10x objectives. Photographic plates were prepared and assembled using Adobe Photoshop.

\section{Tyramide Signal Amplification (TSA) reactions}

To compare the detection sensitivity of fluorescence in situ hybridization and chromogenic in situ hybridization, we also employed the TSA Plus Cyanine 5 Kit (Perkin Elmer NEL745E001KT, TSA Plus Fluorescence Systems, Waltham, MA) to detect the mRNA of UNC5 according to the manufacturer's instructions. Briefly, after biotinlabeled UNC5 probe hybridization and post-hybridization washes, the lamprey brains were blocked in TNB blocking buffer $(0.1 \mathrm{M}$ Tris- $\mathrm{HCl}$, pH7.5, $0.15 \mathrm{M} \mathrm{NaCl}, 0.5 \%$ Blocking Reagent) for 2 hours at RT. The UNC5 mRNA probe was then detected by incubation at $4^{\circ} \mathrm{C}$ overnight in streptavidin- $\beta$-peroxidase (POD) conjugate (Roche, 11089153001) diluted 1:1000 in TNB buffer. Next day the brain tissue was washed 15 min at RT in TNT Buffer (0.1 M Tris-HCl, pH7.5, 0.15 M NaCl, 0.05\% Tween 20) with agitation, and incubated in Cyanine5-labeled tyramide amplification working solution (Cyanine5 plus TSA stock solution 1:50 diluted using 1x Plus Amplification Diluent) for 5 min in the dark at RT. Reaction was stopped by washing in TNT Buffer for $15 \mathrm{~min}$ at RT. Brains were then mounted under Fluroromount-G (eBioscience, 
Citation: Chen J, Laramore C, Shifman M (2015) Triple-Labeling Whole-Mount In Situ Hybridization Method for Analysis of Overlapping Gene Expression in Brain Tissue with High Level of Autofluorescence. J Cytol Histol S3:011. doi:10.4172/2157-7099.S3-011

San Diego, CA) mounting medium, and pictures were taken under a Nikon microscope (Nikon Eclipse 80i) with attached digital camera using $2 \mathrm{x}, 4 \mathrm{x}$ and 10x objectives. Photographic plates were prepared and assembled using Adobe Photoshop.

\section{Results and Discussion}

\section{Autofluorescence in the lamprey brain tissue is the key obstacle to FISH}

Lampreybrain cells displayvery strong endogenous autofluorescence with a broad excitation spectrum (Figure 1), which makes the multicolor FISH method unsuitable. Moreover, our experiments did not reveal autofluorescence suppression by histochemical techniques $\left(\mathrm{CuSO}_{4}\right.$ or SB) (results not shown). Therefore, we developed a new sequential chromogenic triple-labeling in situ hybridization method for detection of digoxigenin/fluorescein/biotin-labeled probes.

\section{Sequential triple colorimetric in situ hybridization of whole- mount lamprey brain}

Alkaline phosphatase substrates, which produce reaction products of different colors, have been well reviewed [6]. We selected AP substrates that produce ethanol-soluble reaction products, such as Fast Red and INT-BCIP, for the sequential colorimetric in situ hybridization labeling. Our third substrate (NBT-BCIP), however, produces an ethanol - insoluble product. As described in the Methods, we mixed together three probes - DCC-DIG, Neogenin-BIO and UNC5-FITC and hybridized them to whole-mount larval lamprey brain tissue (Figure 2). In the first round of detection we employed AP-conjugated anti-DIG antibody to recognize the DCC-DIG probe and Fast Red to develop a red signal (Figure 2B, 2E, 2H). We next used AP-conjugated streptavidin to recognize the Neogenin-BIO probe and the INT/BCIP combination to develop a yellow-brown signal for Neogenin mRNA (Figure 2C, $2 \mathrm{~F}, 2 \mathrm{I})$. In the final detection procedure, we used AP-conjugated antiFITC antibody and the NBT-BCIP combination to develop a purpleblue signal for UNC5 mRNA (Figure 2D, 2G, 2J). Using this sequential triple colorimetric in situ hybridization method, we showed that netrin receptors DCC and UNC-5, and the RGM receptor Neogenin, are coexpressed in two of the identified reticulospinal neurons (I1 and B3) in the lamprey brainstem.

A critical element in our protocol is to completely remove and inactivate the previously applied AP-antibody before incubating the sample with the next AP-conjugated antibody. This was achieved by incubating the whole-mount brain preparation in $0.1 \mathrm{M}$ glycine$\mathrm{HCl}, \mathrm{pH} 2.2,0.1 \%$ Tween-20 (Figure 3). The next important step is to wash away the red or yellow-brown color before developing the next colorimetric stain. This step is essential in order not to interfere with the reaction product generated by the next staining reaction in neurons displaying overlapping or co-localized expression of the targeted molecules. Therefore, after image capture, we used a $50 \%$, $70 \%, 90 \%, 95 \%$ and $100 \%$ ethanol sequence to wash away the color that was present. In general, we prefer a Fast Red - INT/BCIP - NBT/BCIP staining sequence, because the first two stains can be washed away by ethanol (Figure 3B). In contrast, the purple-blue NBT-BCIP precipitate is very stable, even in ethanol. One concern about the usefulness of our sequential wash away method is that it may reduce the sensitivity of the

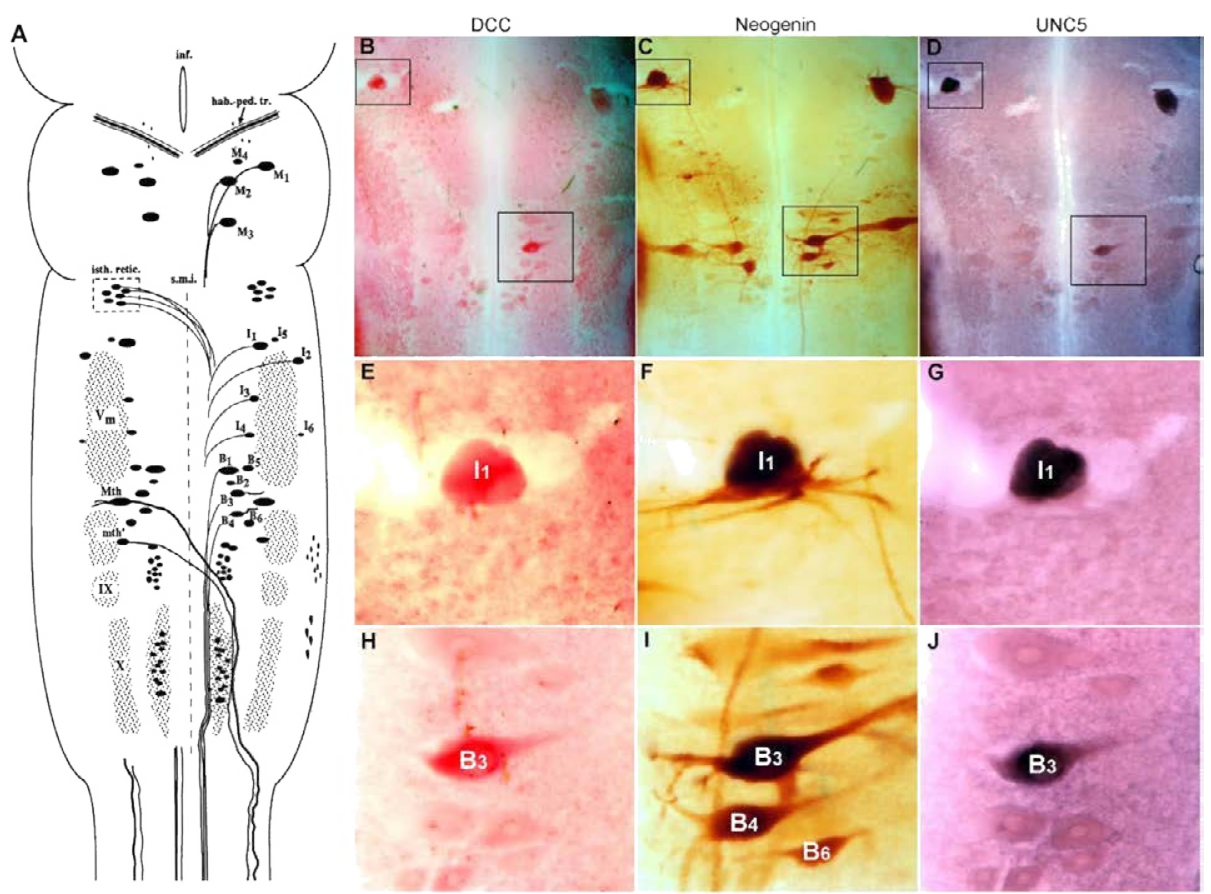

Figure 2: Triple-label in situ hybridization shows that two identified neurons, 11 and B3, express DCC, Neogenin and UNC5 mRNA. (A) Schematic drawing of the large larval lamprey brainstem showing major anatomical features and the locations of identified neurons and neuron groups. I, isthmic; M, mesencephalic; B, bulbar; Mth, Mauthner cell; mth, auxiliary Mauthner cell; hab.-ped. tr., habenulopeduncular tract; inf., infundibulum; isth.retic., isthmic (anterior rhombencephalic) reticulospinal nucleus; s.m.i., sulcus medianus inferior; $\mathrm{Vm}$, trigeminal motor nucleus; IX, glossopharyngeal motor nucleus; $\mathrm{X}$, vagal motor nucleus. The view is from the dorsal (ventricular) surface. (B) DCC mRNA detection by DCC-digoxigenin probe followed by Alkaline Phosphatase (AP)-Fast Red staining. (C) Second step - Neogenin mRNA detection by in situ hybridization with Neogenin-biotin probe and developed with the AP-INT/BCIP system. (D) Third step - UNC5 mRNA detection by in situ hybridization with UNC5-fluorescein probe and developed with AP-NBT/BCIP system. (E-J) Magnifications of the images in the boxes in B, C and D. The large identified reticulospinal neurons I1 and B3 co-express mRNAs for all three receptors: DCC, Neogenin and UNC5. 

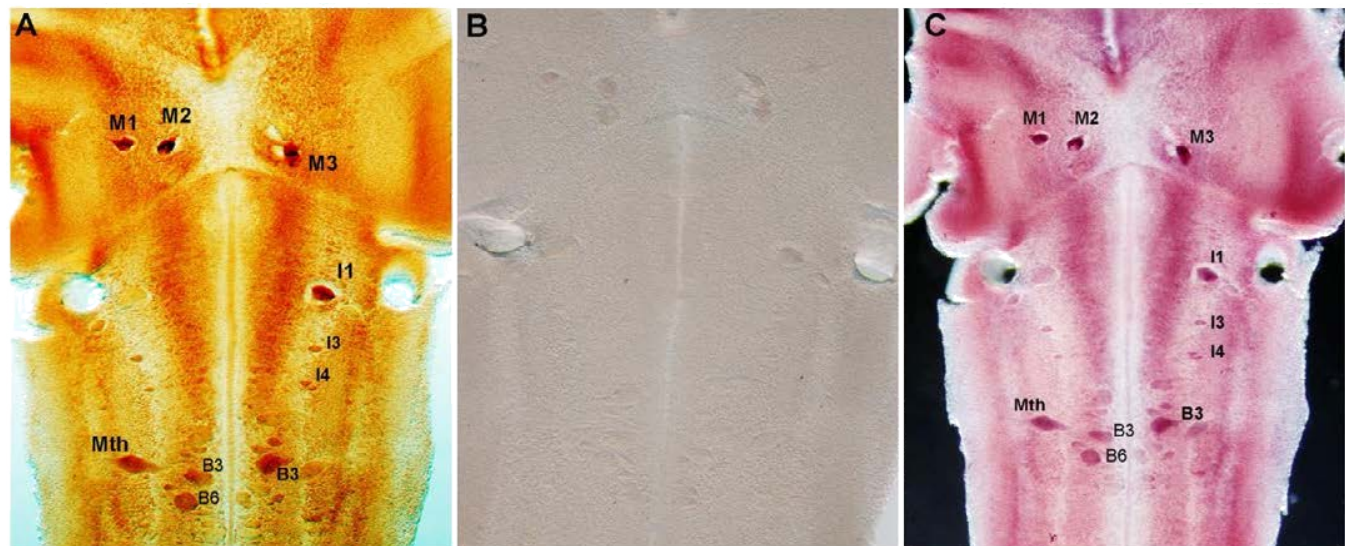

Figure 3: The AP substrates INT/BCIP and NBT/BCIP have comparable sensitivities. (A) In situ hybridization of the whole-mount larval lamprey brainstem with DCC-digoxigenin antisense probe developed with AP-INT/BCIP system. Several large identifiable neurons (M1, M2, M3, I1, I3, I4, Mauthner and B cells) are stained yellow-brown. (B) Lamprey brain after sequential ethanol washes but without glycine-Tris- $\mathrm{HCl}$, $\mathrm{pH} 2.2$ treatment. Note that the previously observed yellow-brown color has been completely washed away. (C) Color development of the same brainstem with NBT/BCIP. Note that all the cells that stain purpleblue when visualized with NBT-BCIP previously stained yellow-brown when visualized by INT-BCIP in A.

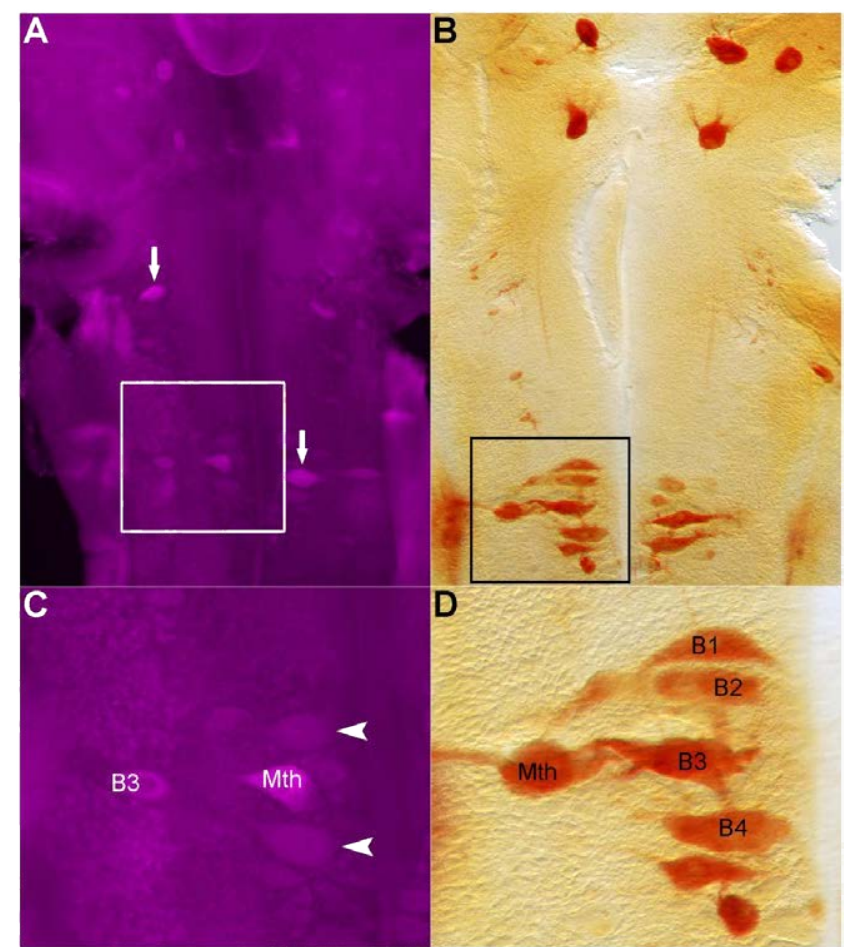

Figure 4: The INT/BCIP chromogenic in situ hybridization method is more sensitive and shows less background staining than the TSA Plus Cyanine 5 Kit. (A) Detection of UNC5 mRNA in lamprey brainstem by fluorescent in situ hybridization (Cyanine5 Tyramide Signal Amplification Kit). Severa identified reticulospinal neurons are labeled (white arrows), but background staining is high. (B) Detection of UNC5 mRNA by chromogenic in situ hybridization using the AP-INT/BCIP method. This method reveals more stained neurons, and background staining is much lower. (C) Enlarged pictures of the images in box in panel A. Some neurons (e.g., Mauthner and B3) are easily distinguished from background staining, but others are more difficult to differentiate with the TSA fluorescent in situ hybridization method (white arrowhead). Mth, Mauthner. (D) High magnification of labeled neurons in box in panel $B$. More bulbar reticulospinal neurons (e.g., $\mathrm{B}_{1}, \mathrm{~B}_{2}, \mathrm{~B}_{3}, \mathrm{~B}_{4}$ ) are positively labeled with the chromogenic AP-INT/ BCIP method than with TSA fluorescent in situ hybridization. Note that non-specific background staining is much lower with chromogenic in situ hybridization. second and third rounds of detection [6]. We ruled out this possibility by conducting the following experiment. We hybridized the wholemount lamprey brain with DCC-DIG probe, used AP-conjugated anti-DIG antibody to recognize it and developed the color reaction with INT/BCIP. As shown in Figure 3A, giant reticulospinal neurons (M1, M2, M3, I1, I3, I4, a Mauthner neuron and several B cells) were positively stained with a yellow-brown color. After capturing the images, we washed the color away with ethanol and did not inactivate the AP enzyme. Instead, we next developed a new color reaction with NBT/ BCIP. As shown in Figure 3C, the location and number of all the cells showing a purple-blue color are the same as the cells showing a yellowbrown color (Figure 3A). Thus, our data show that the sensitivities of AP substrates INT/BCIP and NBT/BCIP are equal (Figure 3A and 3C).

One of the most important characteristics of the in situ hybridization method is its sensitivity and ability to detect mRNA targets that are not abundant. This feature is especially important to us because our experimental goal is to detect mRNA for axonal guidance receptors, which are sparse. Therefore, after we developed the multicolor AP-based chromogenic in situ hybridization system, we compared the sensitivity of AP-INT/BCIP chromogenic in situ hybridization to that of the TSA enhanced FISH Kit (PerkinElmer, TSA Plus Cyanine 5 Kit) using the UNC5-biotin probe. FISH methods based on signal amplification by the TSA reaction are generally considered the most sensitive of the FISH methods; on average their sensitivity is 100 times greater than that of conventional FISH [8-10] (Figure 4). As illustrated in Figure 4A and $4 \mathrm{C}$, although we can see positively labeled Mauthner and B3 neurons using the TSA system, the background level is very high and difficult to distinguish from true labeling. In contrast, when we use the APINT/BCIP reaction system, more neurons are unambiguously labeled (Figure $4 \mathrm{~B}$ and $4 \mathrm{D}$ ). That TSA-amplified FISH is at best only equally as sensitive as NBT-BCIP has been reported previously [29], and other groups have reported FISH sensitivity to be less than that of NBTBCIP [12]. Our data showed that chromogenic in situ hybridization using AP-INT/BCIP is more sensitive than with detection using the POD-TSA amplification system. TSA may fail to detect transcripts that are not abundant because POD activity is rather quickly quenched by excess substrate $[8,30]$. 
Citation: Chen J, Laramore C, Shifman M (2015) Triple-Labeling Whole-Mount In Situ Hybridization Method for Analysis of Overlapping Gene Expression in Brain Tissue with High Level of Autofluorescence. J Cytol Histol S3:011. doi:10.4172/2157-7099.S3-011

\section{Novelty and application of our sequential triple colorimetric in situ hybridization method}

The main research goal of our laboratory is to study co-expression of axonal guidance receptors (DCC, UNC5, Neogenin, PlexinB) in regenerating lamprey neurons. Multicolor FISH detects colocalization of multiple mRNAs in single neurons at high resolution $[11,30,31]$. However, we cannot apply this method because of the high autofluorescence of lamprey brain tissue. The only viable alternative in situ hybridization method is chromogenic in situ hybridization. Multicolor colorimetric in situ hybridization offers good sensitivity and is unaffected by autofluorescence. Many chromogenic multilabeling methods for detection of digoxigenin / biotin / fluoresceinlabeled probes have been developed [5-7,29,32]. Our strategy was first to combine the same alkaline phosphatase with Fast Red, INT/BCIP and NBT/BCIP substrates, which would provide reaction products of different colors. We then captured images immediately after each staining step, inactivated AP and washed away the current color with ethanol before the next round of antibody incubation and staining. This innovation allowed us to avoid one of the main disadvantages of previously reported procedures for multicolor AP- based chromogenic in situ hybridization: inability to distinguish overlapping or co-localized gene expression patterns because they are difficult to differentiate when produced at the same site. For example, the purple-blue BCIP-NBT reaction product can become very dark and obscure the previously obtained Fast Red or INT/BCIP signals [6]. It is difficult to control the optimal reaction time at each step of these procedures, and the final three-color picture may hide information about genes visualized by weaker colors. This consideration is particularly important when studying gene expression of multiple molecules in single neurons. Our method makes it relatively easy to control each step with an appropriate reaction time and to get a clear view of each individual gene represented by a separate color.

Additionally, this sequential triple colorimetric in situ hybridization method also work pretty well on either frozen or paraffin sections of brain/ spinal cord tissue mounted on slides (data not shown). The last but not the least to mention is that, after the first two-round washable in situ hybridization staining, immunochemistry staining for one target protein can also be achieved on the same sample subsequently. This will definitely help expand our method to a wider research field which focus on the distribution and co-expression of different target genes and proteins in the same cell.

\section{Acknowledgments}

We thank Dr. Michael E. Selzer for his careful reading and valuable suggestions. This work was supported by grants from Shriners Research Foundation SHC85310 to MS and Shriners Research fellowship grant SHC-84297 to JC.

\section{References}

1. Rosen B, Beddington R (1994) Detection of mRNA in whole mounts of mouse embryos using digoxigenin riboprobes. Methods Mol Biol 28: 201-208.

2. Hauptmann G, Gerster T (1996) Multicolour whole-mount in situ hybridization to Drosophila embryos. Dev Genes Evol 206: 292-295.

3. Quiring R, Wittbrodt B, Henrich T, Ramialison M, Burgtorf C, et al. (2004) Largescale expression screening by automated whole-mount in situ hybridization. Mech Dev 121: 971-976.

4. Nikitina N, Bronner-Fraser M, Sauka-Spengler T (2009) Whole-mount in situ hybridization on lamprey embryos. Cold Spring Harb Protoc 2009: pdb prot5125.

5. Speel EJ, Ramaekers FC, Hopman AH (1995) Cytochemical detection systems for in situ hybridization, and the combination with immunocytochemistry, 'who is still afraid of red, green and blue?'. Histochem J 27: 833-858.
6. Hauptmann G (2001) One-, two-, and three-color whole-mount in situ hybridization to Drosophila embryos. Methods 23: 359-372.

7. Barroso-Chinea P, Aymerich MS, Castle MM, Perez-Manso M, Tunon T, et al (2007) Detection of two different mRNAs in a single section by dual in situ hybridization: A comparison between colorimetric and fluorescent detection. J Neurosci Methods 162: 119-128.

8. Bobrow MN, Harris TD, Shaughnessy KJ, Litt GJ (1989) Catalyzed reporter deposition, a novel method of signal amplification. Application to immunoassays. J Immunol Methods 125: 279-285.

9. Clay $H$, Ramakrishnan $L$ (2005) Multiplex fluorescent in situ hybridization in zebrafish embryos using tyramide signal amplification. Zebrafish 2: 105-111.

10. Zaidi AU, Enomoto H, Milbrandt J, Roth KA (2000) Dual fluorescent in situ hybridization and immunohistochemical detection with tyramide signal amplification. J Histochem Cytochem 48: 1369-1375.

11. Denkers N, García-Villalba P, Rodesch CK, Nielson KR, Mauch TJ (2004) FISHing for chick genes: Triple-label whole-mount fluorescence in situ hybridization detects simultaneous and overlapping gene expression in avian embryos. Dev Dyn 229: 651-657.

12. Zhou X, Vize PD (2004) Proximo-distal specialization of epithelial transport processes within the Xenopus pronephric kidney tubules. Dev Biol 271: 322-338.

13. Schaaf MM, Schmidt T (2011) In Vivo Single-Molecule Microscopy Using the Zebrafish Model System. In: Sako Y, Ueda M, Cell Signaling Reactions: Springer Netherlands.

14. Zeng Y, Xu J, Li D, Li L, Wen Z, et al. (2012) Label-free in vivo flow cytometry in zebrafish using two-photon autofluorescence imaging. Opt Lett 37: 2490-2492.

15. Dowson JH, Armstrong D, Koppang N, Lake BD, Jolly RD (1982) Autofluorescence emission spectra of neuronal lipopigment in animal and human ceroidoses (ceroid-lipofuscinoses). Acta Neuropathol 58: 152-156.

16. Hillman H, Hussain T, Sartory $P$ (1973) Autofluorescence of isolated unfixed rabbit Deiters' neurons and surrounding neuroglial clamps. Experientia 29 1113-1115.

17. Benson RC, Meyer RA, Zaruba ME, McKhann GM (1979) Cellular autofluorescence--is it due to flavins? J Histochem Cytochem 27: 44-48.

18. Spitzer N, Sammons GS, Price EM (2011) Autofluorescent cells in rat brain can be convincing impostors in green fluorescent reporter studies. J Neurosci Methods 197: 48-55

19. Tsai EC, van Bendegem RL, Hwang SW, Tator CH (2001) A novel method for simultaneous anterograde and retrograde labeling of spinal cord motor tracts in the same animal. J Histochem Cytochem 49: 1111-1122.

20. Zhou XF, Rush RA, McLachlan EM (1996) Differential Expression of the p75 Nerve Growth Factor Receptor in Glia and Neurons of the Rat Dorsal Root Ganglia after Peripheral Nerve Transection. J Neurosci 16: 2901-2911.

21. Lee MS, Kubota K, Iseki H, Shibanai S, Nagae K, et al. (1988) Degenerative changes of the primary trigeminal axons and neurons following infraorbital nerve transection. Anat Anz 165: 351-369.

22. Dowson JH (1982) The evaluation of autofluorescence emission spectra derived from neuronal lipopigment. J Microsc 128: 261-270.

23. Billinton N, Knight AW (2001) Seeing the wood through the trees: a review of techniques for distinguishing green fluorescent protein from endogenous autofluorescence. Anal Biochem 291: 175-197.

24. Schnell SA, Staines WA, Wessendorf MW (1999) Reduction of lipofuscin-like autofluorescence in fluorescently labeled tissue. J Histochem Cytochem 47 719-730.

25. Clancy B, Cauller LJ (1998) Reduction of background autofluorescence in brain sections following immersion in sodium borohydride. J Neurosci Methods 83 97-102.

26. Cowen T, Haven AJ, Burnstock G (1985) Pontamine sky blue: A counterstain for background autofluorescence in fluorescence and immunofluorescence histochemistry. Histochemistry 82: 205-208.

27. Neumann M, Gabel D (2002) Simple method for reduction of autofluorescence in fluorescence microscopy. J Histochem Cytochem 50: 437-439.

28. Szöllösi J, Lockett SJ, Balázs M, Waldman FM (1995) Autofluorescence correction for fluorescence in situ hybridization. Cytometry 20: 356-361. 
Citation: Chen J, Laramore C, Shifman M (2015) Triple-Labeling Whole-Mount In Situ Hybridization Method for Analysis of Overlapping Gene Expression in Brain Tissue with High Level of Autofluorescence. J Cytol Histol S3:011. doi:10.4172/2157-7099.S3-011

Page 7 of 7

29. Hurtado A, Moon LD, Maquet V, Blits B, Jerome R, et al. (2006) Poly (D,L-lactic acid) macroporous guidance scaffolds seeded with Schwann cells genetically modified to secrete a bi-functional neurotrophin implanted in the completely transected adult rat thoracic spinal cord. Biomaterials 27: 430-442.

30. Lauter G, Söll I, Hauptmann G (2011) Two-color fluorescent in situ hybridization in the embryonic zebrafish brain using differential detection systems. BMC Dev Biol 11: 43.
31. Kosman D, Mizutani CM, Lemons D, Cox WG, McGinnis W, et al. (2004) Multiplex detection of RNA expression in Drosophila embryos. Science 305 846.

32. Jowett T (2001) Double in situ hybridization techniques in zebrafish. Methods 23: $345-358$

This article was originally published in a special issue, Cytopathology

handled by Editor(s). Borislav A. Alexiev. Department of Pathology

University of Maryland Medical Center, USA 